\title{
Context Sensitive Fire Protection System
}

\author{
Peter Jankư ${ }^{\mathrm{a}}$, Karel Koplík ${ }^{\mathrm{b}}$, Roman Došek ${ }^{\mathrm{a}}$, Tomáš Dulík ${ }^{\mathrm{a}}$, \\ Michal Bližňák ${ }^{\mathrm{a}}$, Pavel Vařacha ${ }^{\mathrm{a}}$ \\ ${ }^{a}$ Faculty of applied informatics, Tomas Bata University in Zlin, Nad Stranemi 4511, Zlin 760 05, Czech Republic \\ ${ }^{b}$ Faculty of information technology, Brno university of technology in Brno, \\ Bozetechova 1/2, Brno 612 66, Czech Republic
}

\begin{abstract}
This paper deals with design of context sensitive fire protection system, which should be able to detect and localize fire in industrial areas, especially in large factory halls. The "context sensitive" means that the system will be able to detect the fire, but also recognize the fire type and decide whether the detected fire is intended (as a part of standard production process) or it is an accident. Moreover, a remote controlled master stream device is part of the designed system; therefore, the system can directly start fire-fighting. The main feature of this system will be extremely low level of false positive actions and minimal collateral damage to the environment surrounding the fire.
\end{abstract}

Keywords: Fire protection; remote controlled master stream device; fire-fighting
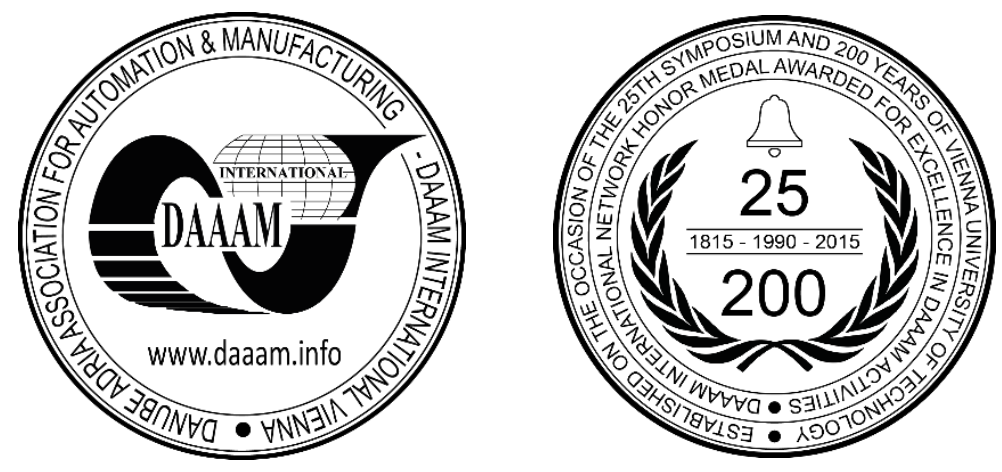

This Publication has to be referred as: Janku, P[eter]; Koplik, K[arel]; Dosek, R[oman]; Dulik, T[omas]; Bliznak, M[ichal] \& Varacha, P[avel] (2016). Context sensitive fire protection system, Proceedings of the 26th DAAAM International Symposium, pp.1025-1031, B. Katalinic (Ed.), Published by DAAAM International, ISBN 978-3-90273407-5, ISSN 1726-9679, Vienna, Austria

DOI: $10.2507 / 26$ th.daaam.proceedings. 144 


\section{Introduction}

The topic of fire protection is discussed in many research papers and research projects as well. Every fire protection system must full fill very strict requirements, but some of them are contradictory. For example, fire detection system must be as fast as possible because unintended fire can cause serious injuries, deaths and material damage. On the other hand, the fire detection system should not trigger false alarm events, because false detection can cause financial losses similar to a real fire. The problem is that many industrial areas use high temperature processes with properties similar to unintended fire as an essential part of their production. To avoid false detection, fire protection system might need more time for evaluating the fire characteristics, but the decision time is limited by the first requirement. [1], [2]

This paper describes a fire protection system based on robotic master stream device(s), which is able to start efficient fire-fighting automatically.

This presupposes that the fire is unambiguously detected and localized. As it is described in section II, conventional methods are seriously inaccurate and/or slow, especially in industrial environment. Therefore, modern methods based on computer vision and stereo-vision should be implemented in smart fire protection systems.

In the next section of this paper, a basic fire detection methods are described including its real features as well as new computer vision methods. In section III-A, the automatic master stream device is discussed including other previously developed devices. The rest of this paper deals with context sensitive fire protection system design. Its internal structure is shown and interaction between parts is explained.

The described system brings new approach in combination of various fire detection methods and automatic master stream devices in order to compose smart sensitive fire protection system. The main aim is to meet demanding requirements for industrial applications

\section{Fire detection methods}

In this section, commonly used sensors will be described and the key principles of computer-vision based algorithm used in more advanced systems will be depicted.

\subsection{Smoke detectors}

Smoke detector is a device that can sense smoke. Most smoke detectors work either by optical detection or by chemical process or by both to increase the sensitivity. Optical smoke detectors emit a beam of infrared or ultraviolet light which is either received by a separate device or reflected back. This is useful when detecting slow, smouldering fires [3].

The ionization smoke detectors use a radioisotope to produce ionization in air between two charged electrodes, creating a passage of a small electric current. When a difference due to smoke is detected, the alarm is triggered. This type of detector is very good when detecting the flaming stage of fire.

As it was said previously, smoke detectors are fast but sometimes have high false alarm ratio. It is a known fact that smoke detectors often get turned off because of that.

\subsection{Heat detectors}

Heat detector is a device that is designed to detect presence of fire by checking the temperature of its surrounding medium (e.g. air). Today, these devices can be set to trigger alarm when the temperature suddenly rises (rate-of rise detector) or when it rises above the fixed value. The thermal conductivity of the temperature sensitive component determines how big the thermal lag of each heat detector is.

Heat detectors detect fire when the temperature is very high which means that the fire already spread around the room / hall. They get installed at places where smoke detectors can be of no use due to common false alarms (e.g. kitchen) [4] but are, nevertheless, much slower. The accuracy, though, is very high.

\subsection{Fire gas detectors}

Fire gas detector is a device detecting products of combustion in the air. Mostly, it's carbon monoxide (CO) fire detectors which contain an electronic circuit and an electrochemical cell that produces a small electrical current in the presence of carbon monoxide. The electronic circuit is calibrated to a normal range of atmospheric carbon monoxide. When the concentration of the carbon monoxide increases the value of current produced by the cell also rises which then triggers the alarm.

Carbon monoxide detectors cannot replace smoke detectors but are often combined with them. However, as a fire detector they give false alarms if the level of $\mathrm{CO}$ is not caused by fire. Fire CO Detectors are more sensitive than ordinary CO Detectors. [5] 


\subsection{Optical flame detector}

Optical flame detector is a device detecting presence of smoke or flames from illumination. There are many different types (ultraviolet, infrared, near infrared array and other). They are fast but false alarms can be triggered by hot surfaces and background thermal radiation in the area.

IR flame detectors sometimes come in a form of a thermographic camera (see Fig. 1). IR smoke detectors can be realized as an end-to-end beam detector [6]. There are other types which combine more IR wavelength ranges or both IR and UV ranges [7].

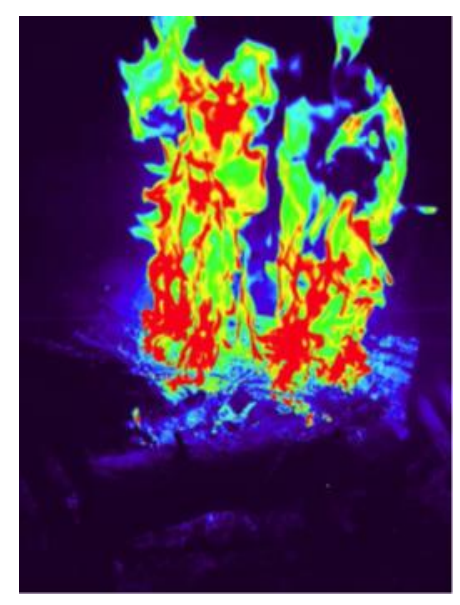

Fig. 1. Fire Captured by Thermal Camera

\subsection{Computer vision methods}

The most efficient computer-vision-based algorithms used today can be generalized to one abstract algorithm. For the sake of this paper, it will be referred to as Color-Motion-Features (CMF) Algorithm.

The CMF Algorithm first detects fire-colored pixels and motion and then investigates fire-like features of suspicious regions. Feature checking consists of more sub-algorithms which are put together to calculate the final decision function.

The preprocessing may consist of:

- Compensation of camera motion

- Color normalization

It is better if the camera is static but it does not necessarily have to be. There are basically two possibilities:

- Camera is moving and the motion compensation slightly distorts the result

- Multiple cameras are used to monitor different directions

Colour normalization is also not mandatory but can improve the results if there is a significant change of contrast during monitoring due to daytime proportions of light etc. The main procedure works as a decision tree and consists of following steps:

- Creation of a fire-colored pixels binary map

- Creation of a motion-containing pixels binary map

- Investigation of suspicious areas

The computer vision methods are constantly evolving and are being increasingly deployed in existing camera systems.[10,11]

\section{Implementation of fire protection system}

As could be seen in figure 2, the whole system is divided into three layers. Devices for HI-level communication are placed on the top system layer, main computation units are placed into middle layer and all smart sensors, analog sensors and cameras are placed in the bottom layer. 


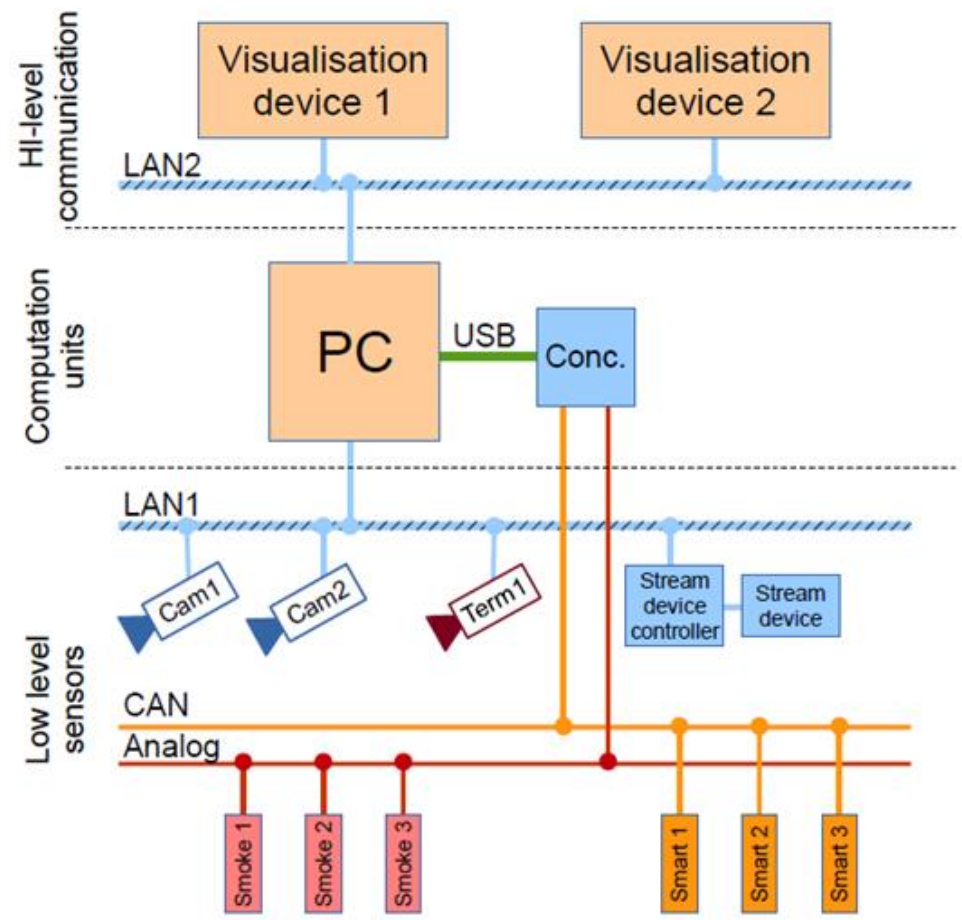

Fig. 2. System architecture

The hi-level communication layer uses standard Ethernet network for communication. It is possible due to autonomous function of whole fire protection system. A standard tablets and computers can be used as visualization device; moreover, this system can be connected into existing security systems. Thanks to this all actual information are simply presented to the security operator. In semiautomatic mode the system is only detecting fires, but cannot start firefighting automatically - the master stream device(s) can be triggered by operator using control station SW running on a visualization device.

The small factor industrial computer is used as a main computation unit. If it is appropriate to the environment conditions, for example in offices, a standard personal computer can be used instead of industrial computer. To reduce a time required for processing of single frame, it is possible to use parallelized vision algorithms running on modern graphic cards using CUDA or OpenCL. Consequently, higher frame rate improves accuracy of entire detection process.

Because of problems with computer connectivity (CAN bus and analog loops required for connecting the sensors), the new device called concentrator was developed. It is placed in middle system layer and it is connected to the computer by using USB or Ethernet. As described in figure 2, the concentrator provides CAN and analog loop connectivity for the computer.

The main part of the fire protection system - set of sensors - is placed in the bottom system layer. As described before, sensors can be connected by using three independent buses. Commonly used smoke, heat and fire gas detectors are connected to the concentrator by using standard analog loops. These sensors are only able to detect a fire, but without fire type detection. Moreover, in order to precise fire type detection, smart sensors are being developed now. They are able to provide more information about detected event, for example the type of detected gas and/or the exact location of the fire. These smart detectors are connected by using CAN bus. [8], [9]

The main feature of this system (fire type detection) is performed on computer vision base. Therefore, cameras and thermo-cameras are standard part of sensor set. They have to be placed in sufficient density and their fields of view must be overlapped. Thanks to this, the starting fire can be easily localized and automatic stream device can be pointed to it. Communication between cameras, stream device controller and main computation unit is performed by using LAN or industrial LAN network.

\subsection{Remote controlled master stream device}

The stream device can be seen in figure 3. This device is supplemented by a camera allowing more precision fire targeting. This camera is not used for fire detection due to its small video quality; moreover, after the start of streaming, its field of view is drown by streamed medium. For this reason, the focus clarification by using this camera can only be performed without stream. Moreover, thanks to its control unit, the stream device can be controlled remotely by using LAN network. The integration of automatic stream device into developing system allows immediately start with firefighting. 


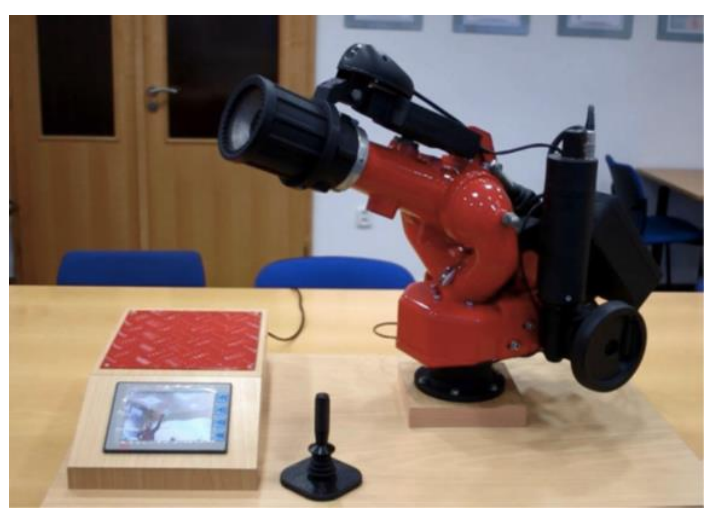

Fig. 3. Automatic master stream device

\subsection{THTronic - visualization device}

THTronic was originally developed as the main on-board computer for controlling and monitoring functions of fire engine trucks. THTronic hardware was designed to achieve industrial working temperature ranges and high mechanical resistance. The ARMv7 is selected as the main computation core. Therefore, this device is able to use standard Linux distributions. It is very suitable for controlling the master steam device and/or for fire protection system status visualisation.

\subsection{Concentrator}

A concentrator is a hardware device, which is used to provide CAN bus connection and standard analog loop connection to the main computer unit. It is based on 32bit ARM processor manufactured by STMicro and bare-metal firmware written in C. The FreeRTOS is used in the firmware; thanks to this the required reaction time is guaranteed.

\subsection{Smart sensors}

As was written in previous sections, the smart fire detector is developed in order to detect fire and fire distinguish from other processes such as welding. For this purpose the PIR sensor was selected as a base detector. The signal measure by PIR sensor can be seen in figure 4 and in figure 5. Signals are ten seconds long samples representing various situations.

The PIR sensor signal is generated on differential base - there are two detecting areas on it and the output voltage level is composed as a difference between voltage levels came from these areas. Therefore, when the same radiation comes to these areas the output signal is nearly constant regardless of its level. This situation is described by the green line (small idle signal comes to the sensor) in figure 4. On the other hand, yellow, orange and blue lines represent the radiation of fire. It can be seen that the amount of fire (amount of flames) causes interferences on the output signal. By using of frequency analysis and amplitude analysis the fire can be detected especially in advanced states of fire.

The radiation of welding process (purple line) in comparison with fire radiation (blue line) is shown in figure 5 As can be seen in this figure the welding radiation consists nearly the same low level of disturbance as an environment radiation level measured in idle state. The peaks in purple line represents the initial/final moments of welding. The stable parts of purple line represents continuous welding process, where all sensor parts are nearly same saturated by welding radiation (regardless its high level). Thanks to this characteristic, the fire can be distinguished from the welding process. A similar principle can be used in distinguishing other processes similar to combustion.

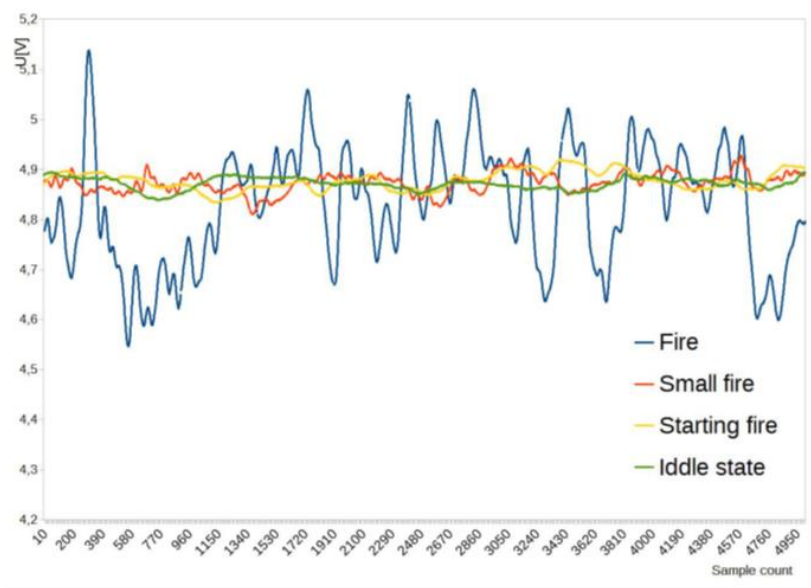

Fig. 4. The signal of three levels of fire in comparison with idle state measured by PIR sensor. 


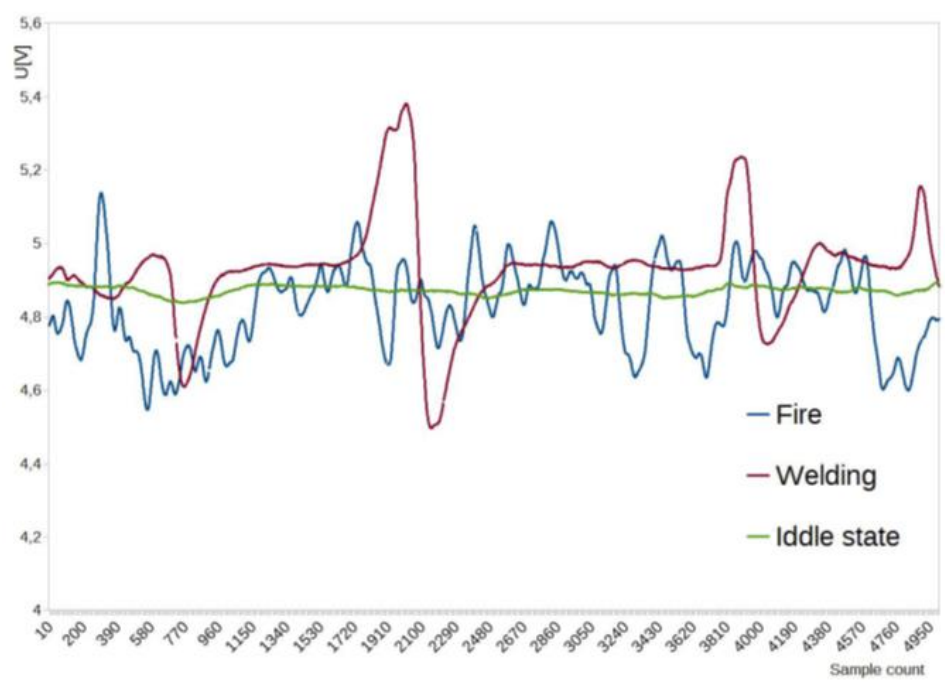

Fig. 5. the signal of fire and welding in comparison with idle state measured by PIR sensor

\section{Detection process}

The fire detection process is very complex. It is performed by using set of criteria applied in serial. Moreover, more sophisticated methods such as Hidden Markov model, neural networks and etc. are used to determine whether or not the fire is detected. This process is actually under development and testing and will be published in further papers.

\section{Conclusion}

The problematic of fire protection systems in industrial areas is very complex problem. These systems has to meet set of requirements as a precise fire detection, a fire type recognition and very low level of false detections.

The design of fire protection system was described in this paper; moreover, basic fire detection methods were discussed and all system parts were presented including system architecture. The main aim of this system is to compose various number of different sensors in order to detect and locate fire. As a part of this system, the set of remote controlled master stream devices were used.

Moreover, PIR sensor fire measurement and its using as a fire detector was described in the end of the paper. The result of this research is an architecture description including special PIR sensor and other HW devices.

As was written before the system components have been developed and tested and/or they are currently under development. Designed fire protection system as a whole is currently being compiled and integrated.

Future work is aimed to physical construction of fire protection system prototype, which was described in this paper. Therefore, its more precise analysis including statistic value of false detection level will be presented in future research papers. Moreover, the concrete parameters and measurements of special PIR sensor will be next part of future research.

\section{Acknowledgements}

This paper is supported by the Internal Grant Agency at TBU in Zlin, Project No. IGA/FAI/2015/059 and by the European Regional Development Fund under the project CEBIA-Tech No. CZ.1.05/2.1.00/03.0089 and by the Technology Agency of the Czech Republic as a part of the project called TA03010724 AV and EV LED liminaire with a higher degree of protection.

\section{References}

[1] F. Guan-lin, "Design of fire alarm system based on multi-source information fusion," in 2010 3rd International Conference on Advanced Computer Theory and Engineering (ICACTE), vol. 5, Aug. 2010, pp. V5-269-V5-272.

[2] H. Hu, G. Wang, Q. Zhang, J. Wang, J. Fang, and Y. Zhang, "Design wireless multi-sensor fire detection and alarm system based on ARM," in 9th International Conference on Electronic Measurement Instruments, 2009. ICEMI '09, Aug. 2009, pp. 3-285-3-288.

[3] W. Kuffner, "Method of determining smoke detector spacing in high ceiling applications," Master thesis, Carleton University., 2009.

[4] E. Electronics, "Model Ei 103C Heat Alarm," http://www.safelincs.co.uk/templates safelincs/files/datasheets/14 Ei103C-Datasheet.pdf, 2015, datasheet. 
[5] Apollo, "CO Fire Detectors," http://www.apollo-fire.co.uk/media/57009/ pp2095-co-fire-detectors-the-facts- issue6 locked locked-2-.pdf, 2013.

[6] Apollo, “Conventional End-to-End Optical Beam Detector,” http://www.apollo-fire.co.uk/media/2717370/pp2471 conventional end-to-end optical beam detector issue 1 locked.pdf, 2013.

[7] Apollo , "Flame Detector Applications," http://www.apollofire.co.uk/media/938575/pp2409\%20flame\%20detector\%20applications\%20pin\%20issue\%202.pdf, 2010.

[8] F. Li, L. Wang, and C. Liao, "CAN(Controller Area Network) Bus Communication System Based on Matlab/Simulink," in 4th International Conference on Wireless Communications, Networking and Mobile Computing, 2008. WiCOM '08, Oct. 2008, pp. 1-4.

[9] J. Cui, D. Huang, Z. Wang, and D. Qin, "Implementation of NetworkSmart Sensors for Ultraviolet Fire Detector," in Proceedings of the 2006 IEEE International Conference on Mechatronics and Automation, Jun.2006, pp. 23882392.

[10] P. Foggia, A. Saggese, and M. Vento, "Real-Time Fire Detection for Video-Surveillance Applications Using a Combination of Experts Based on Color, Shape, and Motion," IEEE Transactions on Circuits and Systems for Video Technology, vol. 25, no. 9, pp. 1545-1556, Sep. 2015.

[11] M. Mueller, P. Karasev, I. Kolesov, and A. Tannenbaum, “Optical Flow Estimation for Flame Detection in Videos," IEEE Transactions on Image Processing, vol. 22, no. 7, pp. 2786-2797, Jul. 2013. 